\title{
Clareamento dental de consultório e sensibilidade: relato de caso
}

In-office and at-home tooth bleaching and sensitivity: case report

Blanqueamiento dental en el consultorio y sensibilidad de los dientes: reporte de caso

Edivan Ilton Dantas da COSTA ${ }^{1}$

Thales de Queiroz LOPES

José Henrique de Araújo $\mathbf{C R U Z}^{2}$

Gymenna Maria Tenório GUÊNES ${ }^{3}$

Luanna Abílio Diniz Melquíades de MEDEIROS $^{3}$

Elizandra Silva da PENHA ${ }^{3}$

Manuella Santos Carneiro de ALMEIDA ${ }^{3}$

Camila Helena Machado da Costa FIGUEIREDo ${ }^{3}$

'Graduação em Odontologia, Universidade Federal de Campina Grande, Unidade Acadêmica de Ciências Biológicas, 58708-110 Patos - PB, Brasil

'Graduado em Odontologia, Universidade Federal de Campina Grande, Unidade Acadêmica de Ciências Biológicas, 58708-110 Patos - PB, Brasil

${ }^{3}$ Professora Doutora, Curso de Odontologia da Universidade Federal de Campina Grande, Unidade Acadêmica de Ciências Biológicas 58708-110 Patos - PB, Brasil

\section{Resumo}

Os tratamentos estéticos na odontologia estão sendo bastante procurados na atualidade, entre eles o clareamento dental se destaca. Desta forma, o objetivo do presente estudo foi relatar um caso clínico de clareamento dental em consultório e discutir os aspectos relacionados à alteração de cor, bem como à sensibilidade causada pelo agente. Paciente do sexo masculino, 26 anos, leucoderma procurou atendimento odontológico da Universidade Federal de Campina Grande (UFCG) queixando-se da cor amarelada dos seus dentes. Sistemicamente saudável e com boa condição de saúde bucal, após exame intra-oral foi indicado clareamento dental em consultório com Whiteness HP 35\%. O tratamento proposto foi iniciado na sessão seguinte com profilaxia sob uso de pedra pomes, água e escova de robinson, com posterior aplicação de barreira gengival TopDam e aplicação do gel clareador na proporção 3:1 de peróxido de hidrogênio e espessante, respectivamente, sendo realizado uma nova aplicação a cada 15 minutos, totalizando 3 aplicações em cada sessão, uma sessão semanalmente. Repetindo-se o protocolo recomendado, na terceira sessão foi realizada uma quarta aplicação do gel clareador topicamente nos quatro caninos, uma vez que estes se encontravam mais amarelados. Assim, a mudança de cor ocorreu da escala A3 para A1. O paciente foi submetido a responder dois questionários, o teste de sensibilidade pela escala de Wong e Baker, e o de satisfação do indivíduo em relação à estética do próprio sorriso. Ao término do tratamento, o grau de desconforto de sensibilidade após as sessões foi caracterizando como um desconforto que dói muito, mas perdurando apenas nas primeiras $24 \mathrm{~h}$, e alto nível de satisfação depois do tratamento. Portanto, o clareamento dentário é um procedimento simples, minimamente invasivo e com excelentes resultados estéticos.

Descritores: Clareamento Dental; Sensibilidade da Dentina; Estética Dentária.

\section{Abstract}

Cosmetic treatments in dentistry are currently being sought after, among them tooth whitening stands out. Thus, the aim of the present study was to report a clinical case of dental bleaching in the office and discuss the aspects related to color change, as well as the sensitivity caused by the agent. Male patient, 26 years old, leucoderma sought dental care from the Federal University of Campina Grande (UFCG) complaining about the yellowish color of his teeth. Systematically healthy and in good oral health condition, after oral examination, dental whitening was indicated in the office with Whiteness HP $35 \%$. The proposed treatment was initiated in the next session with prophylaxis using pumice stone, water and robinson brush, with subsequent application of TopDam gingival barrier and application of the bleaching gel in a 3: 1 ratio of hydrogen peroxide and thickener, respectively. one new application every 15 minutes, totaling 3 applications in each session, one session weekly. Repeating the recommended protocol, in the third session a fourth application of the bleaching gel was performed topically on the four canines, since they were more yellowish. Thus, the color change occurred from A3 to A1 scale. The patient was submitted to answer two questionnaires, the sensitivity test by the Wong and Baker scale, and the individual's satisfaction regarding the aesthetics of the smile itself. At the end of the treatment, the degree of sensitivity discomfort after the sessions was characterized as a discomfort that hurts a lot, but lasting only for the first 24 hours, and a high level of satisfaction after treatment. So tooth whitening is a simple procedure, minimally invasive and with excellent aesthetic results.

Descriptors: Tooth Bleaching; Dentin Sensitivity; Esthetics, Dental.

\section{Resumen}

Actualmente se buscan tratamientos cosméticos en odontología, entre los que destaca el blanqueamiento dental. Por lo tanto, el objetivo del presente estudio fue informar un caso clínico de blanqueamiento dental en el consultorio y discutir los aspectos relacionados con el cambio de color, así como la sensibilidad causada por el agente. Un paciente masculino de 26 años, leucoderma, buscó atención dental en la Universidad Federal de Campina Grande (UFCG) quejándose del color amarillento de sus dientes. Sistemáticamente saludable y en buen estado de salud bucal, después del examen oral, el blanqueamiento dental se indicó en la oficina con blancura HP 35\%. El tratamiento propuesto se inició en la próxima sesión con profilaxis con piedra pómez, agua y cepillo robinson, con la aplicación posterior de la barrera gingival TopDam y la aplicación del gel blanqueador en una proporción 3: 1 de peróxido de hidrógeno y espesante, respectivamente. una nueva aplicación cada 15 minutos, un total de 3 aplicaciones en cada sesión, una sesión semanal. Repitiendo el protocolo recomendado, en la tercera sesión se realizó una cuarta aplicación del gel blanqueador por vía tópica en los cuatro caninos, ya que eran más amarillentos. Por lo tanto, el cambio de color ocurrió de A3 a escala A1. El paciente fue enviado a responder dos cuestionarios, la prueba de sensibilidad de la escala de Wong y Baker, y la satisfacción del individuo con respecto a la estética de la sonrisa en sí. Al final del tratamiento, el grado de incomodidad de sensibilidad después de las sesiones se caracterizó como una molestia que duele mucho, pero que dura solo las primeras 24 horas, y un alto nivel de satisfacción después del tratamiento. Por lo que el blanqueamiento dental es un procedimiento simple, como mínimo invasivo y con excelentes resultados estéticos.

Descriptores: Blanqueamiento de Dientes; Sensibilidad de la Dentina; Estética Dental.

INTRODUÇÃO

A busca pelo sorriso perfeito faz do clareamento dentário uma constante na sociedade atual, o que o torna um procedimento bastante requisitado. Desta forma, o clareamento dentário é um tratamento conservador, simples e mais comumente usado 
por clínicos para se obter um sorriso esteticamente agradável. É considerada uma técnica não invasiva que possibilita ao cirurgião dentista corresponder às expectativas dos pacientes em busca de dentes mais claros. Desta forma, o clareamento dentário pode ser definido como um tratamento conservador, onde as substâncias químicas são capazes de se difundirem através da superfície dental liberando radicais livres que oxidam pigmentos orgânicos da estrutura dos dentes, clareando o elemento dentário ${ }^{1,2}$.

O clareamento dentário pode ser realizado de três formas: pelo cirurgião dentista no consultório, realizado pelo próprio paciente, em casa, ou a associação de ambos os tratamentos. O clareamento caseiro consiste na confecção de uma moldeira, para que o próprio paciente faça a aplicação de gel clareador, de acordo com a instrução do dentista. Já o clareamento realizado em consultório, por sua vez, é realizado em âmbito clínico, sendo necessária a intervenção do dentista em todo o tratamento, permitindo uma resposta rápida, pois 0 agente clareador, que pode ser 0 Peróxido de Hidrogênio, é usado em maior concentração $0^{3,4}$.

Assim, o clareamento visa devolver a harmonia estética, tendo como grande vantagem a conservação da estrutura dentária, além de ser um procedimento que possibilita corresponder às expectativas dos pacientes ${ }^{5}$.

Desta forma, o presente estudo tem como propósito relatar um caso clínico de clareamento dental em consultório e discutir os aspectos relacionados à alteração de cor, bem como à sensibilidade causada pelo agente.

CASO CLÍNICO

Paciente gênero masculino, 26 anos de idade, compareceu à Clínica Escola de Odontologia da Universidade Federal de Campina Grande (UFCG), campus Patos, queixando-se da estética do seu sorriso. Após avaliação clínica inicial, constatou-se apenas coloração amarelada dos seus dentes, sobretudo dos caninos (Figura 1). Logo, planejou-se para o paciente um clareamento dental em consultório sob o uso do agente clareador Whiteness HP 35\% (FGM, Joinville $\mathrm{SC}$, Brasil). Na sessão seguinte, seguiu-se 0 protocolo estético com clareamento em consultório sob o uso do gel clareador em três sessões, uma vez por semana durante três semanas. Iniciou-se o procedimento com profilaxia com pedra pomes e água utilizando escova de Robinson (Microdont ${ }^{\circledR}$, São Paulo, $\mathrm{SP}$, Brasil) e posterior verificação na escala de cores Vita (Vitapan® Classical), classificando-se em A3 (Figura 2).

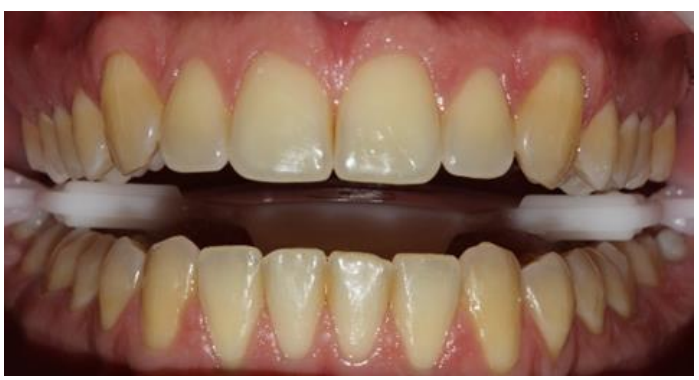

Figura 1: Foto intrabucal frontal sem comparação com a escala de cores

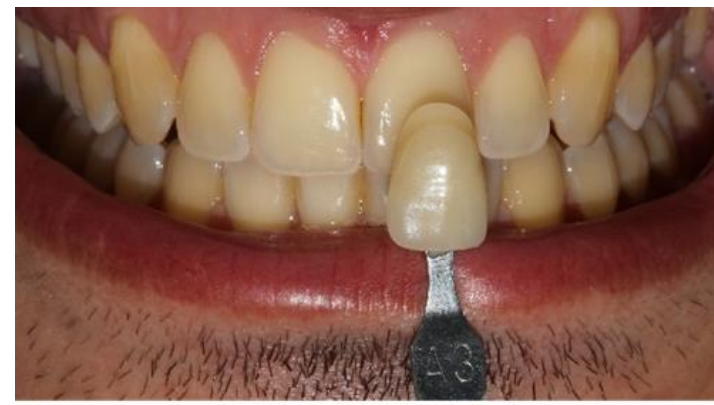

Figura 2: Foto intrabucal frontal do paciente, comparando com escala de cores Vita, que corresponde a cor inicial do caso.

Aplicou-se a barreira gengival top dam (FGM, Whiteness HP, Brasil) de segundo prémolar direito (15) a segundo pré-molar esquerdo (25) superior e inferior (35 ao 45) e inseriu-se o gel clareador de peróxido de hidrogênio a $35 \%$ (Whiteness HP, FGM, Brasil) na face vestibular dos elementos dentários. Foram feitas nesta sessão 3 aplicações, do gel clareador, na proporção de 3:1 de peroxido de hidrogênio e espessante, respectivamente. Cada uma com duração de 15 minutos, até que o agente clareador mudasse totalmente de cor (Figura 3).

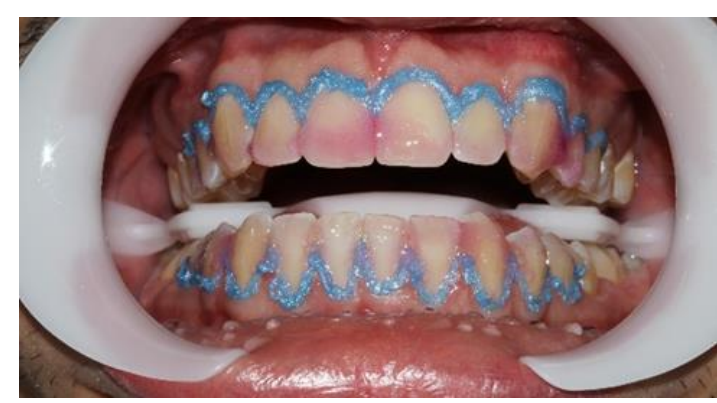

Figura 3: Foto do gel clareador sendo aplicado nos dentes do paciente, utilizando Top Dam e Arcflex

Repetiu-se o protocolo nas demais sessões, e na última sessão, realizou-se uma quarta aplicação de 15 minutos apenas nos quatro caninos $(13,23,33$ e 43), uma vez que estes possuíam intensa cor amarelada e por fim, realizou-se um polimento das superfícies clareadas sob uso de disco de feltro Diamond Flex (FGM) e pasta polidora Diamond Excel (FGM) (Figura 4). Após cada sessão clínica de clareamento dental, empregou-se o questionário 
que registra a sensibilidade dentária de acordo com a escala de classificação de sensibilidade adotada na pesquisa de Wong e Baker ${ }^{6}$, que possui números que variam entre zero a dez, do qual zero corresponde à ausência de sintomatologia dolorosa e dez à bastante sintomatologia dolorosa, como mostra na (Figura 5). Os resultados sobre sensibilidades estão expressos na Tabela 1, conforme o relato do paciente frente à sensibilidade durante $\mathrm{e}$ após as sessões clareadoras.

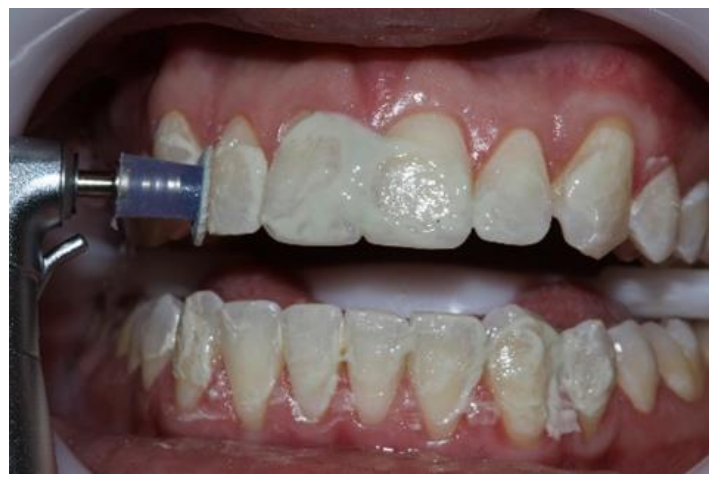

Figura 4: Polimento dos elementos dentários, após feito o clareamento

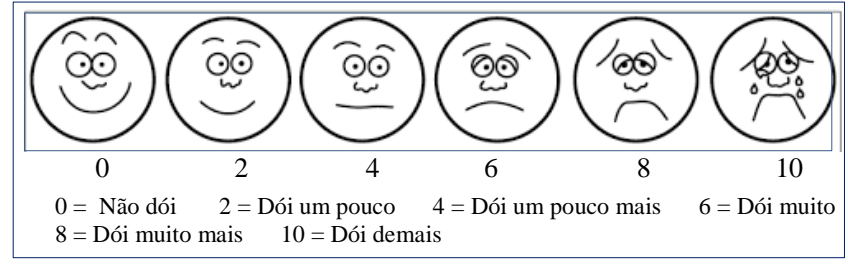

Figura 5: Wong-Baker - Escala para medir sensibilidade (Fonte: Wong e Baker ${ }^{6}$ ).

Tabela 1: Representação da sensibilidade dentária

\begin{tabular}{|ccc|}
\hline & \multicolumn{2}{c|}{ Sensibilidade } \\
\hline $\mathbf{1}^{\mathbf{a}}$ Sessão & $\begin{array}{c}\text { Sensibilidade antes das 12 primeiras horas } \\
\text { e perdurando por um tempo de 12 horas }\end{array}$ & $\begin{array}{c}\text { Score } \\
\text { Dói um pouco } 2\end{array}$ \\
\hline $\mathbf{2}^{\mathbf{a}}$ Sessão & $\begin{array}{c}\text { Sensibilidade após a sessão clareadora } \\
\text { perdurando por um tempo de 24 horas }\end{array}$ & $\begin{array}{c}\text { Score } 6 \\
\text { Dói muito }\end{array}$ \\
\hline $\mathbf{3}^{\mathbf{a}}$ Sessão & $\begin{array}{c}\text { Sensibilidade após a sessão clareadora } \\
\text { perdurando por um tempo de 24 horas }\end{array}$ & $\begin{array}{c}\text { Score } 4 \\
\text { Dói um pouco mais }\end{array}$ \\
\hline
\end{tabular}

Quando indagado sobre em quais momentos do dia-a-dia o paciente sentiu sensibilidade, ele relatou que ao falar, respirar pela boca e quando consumia alimentos gelados. Quanto ao tipo de dor sentida, o paciente relatou que era do tipo lancinante e de passageira duração, nos elementos $31,32,41$ e 42. Também apresentou leve sensibilidade nos caninos (13 e 23) e nenhuma sensibilidade nos demais elementos.

Segundo os resultados obtidos do questionário e o seu score, a maior sensibilidade relatada pelo paciente correspondeu a segunda sessão do clareamento, com "score 06: dói muito". Já as demais sessões não foram significantes segundo seu relato. Após as três sessões, verificou-se mais uma vez a cor final através da escala de cores, e notou-se que a mudança de cor ocorreu da escala A3 para A1 (Figura 6), constatando-se a alteração estética positiva de cores e a satisfação do paciente.

Além disso, também foi aplicado um questionário de satisfação do indivíduo em relação à estética do próprio sorriso Silva ${ }^{7}$ uma vez antes do tratamento clareador ser iniciado e uma vez depois, após a conclusão do clareamento. Os dados foram expressos na Tabela 2.

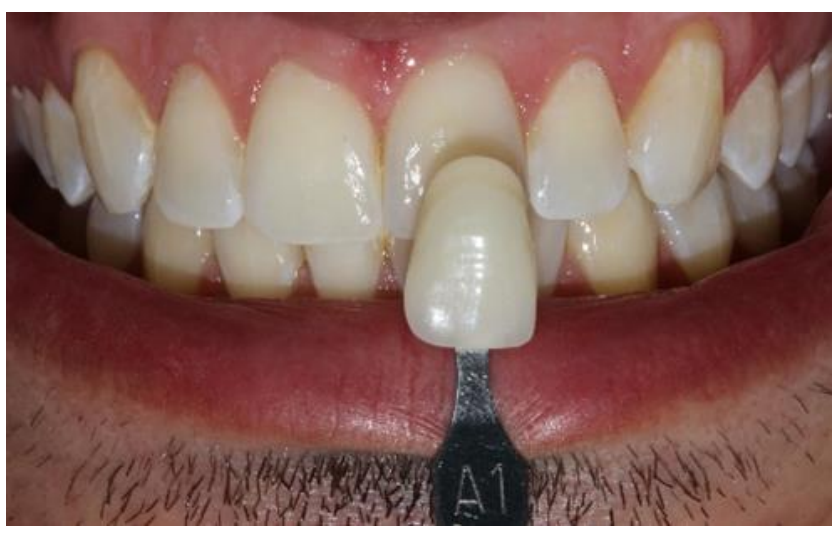

Figura 6: Foto intrabucal comparando com a escala de cores compatível com a cor final, obtida após o clareamento

Tabela 2. Avaliação da percepção estética do sorriso

\begin{tabular}{|c|c|c|}
\hline \multicolumn{3}{|c|}{ Avaliação da percepção estética do sorriso segundo Silva7 } \\
\hline Você é satisfeito(a) com o seu sorriso? & Antes & Depois \\
\hline Muito satisfeito & & $\mathrm{X}$ \\
\hline \multicolumn{3}{|l|}{ Satisfeito } \\
\hline \multicolumn{3}{|l|}{ Pouco satisfeito } \\
\hline Insatisfeito & $\mathrm{X}$ & \\
\hline $\begin{array}{l}\text { Quando você sorri, sente vergonha em mostrar seus } \\
\text { dentes? }\end{array}$ & Antes & Depois \\
\hline Não & & $\mathrm{x}$ \\
\hline \multicolumn{3}{|l|}{ Um pouco } \\
\hline Sim & $\mathrm{X}$ & \\
\hline $\begin{array}{l}\text { Você se sente seguro e confiante em relação ao seu } \\
\text { sorriso }\end{array}$ & Antes & Depois \\
\hline Não & $\mathrm{x}$ & \\
\hline \multicolumn{3}{|l|}{ Um pouco } \\
\hline Sim & & $\mathrm{X}$ \\
\hline De o a 10 que nota você daria para o seu sorriso? & Antes & Depois \\
\hline Nota & 5 & 9,5 \\
\hline $\begin{array}{l}\text { Você considera seu sorriso semelhante ao das outras } \\
\text { pessoas? }\end{array}$ & Antes & Depois \\
\hline \multicolumn{3}{|l|}{ Sim } \\
\hline Não, considero melhor & & $\mathrm{X}$ \\
\hline Não, considero pior & $\mathrm{x}$ & \\
\hline $\begin{array}{l}\text { Você considera seu sorriso uma importante } \\
\text { ferramenta para as relações interpessoais? }\end{array}$ & Antes & Depois \\
\hline Sim & $\mathrm{x}$ & $\mathrm{X}$ \\
\hline \multicolumn{3}{|l|}{ Não } \\
\hline $\begin{array}{l}\text { Você realizaria algum tratamento odontológico para } \\
\text { fins exclusivamente estéticos? }\end{array}$ & Antes & Depois \\
\hline Sim & $\mathrm{x}$ & \\
\hline Não & & $\mathrm{X}$ \\
\hline
\end{tabular}

DISCUSSÃO

Cada vez mais o clareamento dental vem se tornando um procedimento muito requisitado pelos pacientes, por ser um método 
que proporciona melhoria na estética do sorriso sem ser invasivo. Pela alta procura e o grande índice de sucesso, os fabricantes tentam melhorar cada vez mais a composição dos agentes clareadores, tornando-os mais eficazes e o resultado mais rápido, satisfazendo ainda mais o paciente ${ }^{8}$.

De acordo com Roberto et al. ${ }^{9}$, o clareamento dental possui várias formas de tratamento, dentre elas apresentam-se a técnica caseira e o realizado em consultório. No tratamento realizado em consultório, é comum o uso de concentrações de peróxido de hidrogênio que variam de $25 \%$ a $50 \%$ e peróxido de carbamida de $35 \%$, com ou sem fontes de luz, controlado pelo dentista.

Para Barbosa et al. ${ }^{10}$ e Soares et al. ${ }^{4}$, o clareamento em consultório permite uma resposta rápida, pois o agente clareador é usado em maior concentração. Geralmente, o Peróxido de Hidrogênio é usado na concentração de $35 \%$, sendo mais indicado para pequenos grupos dentais. Comumente, em apenas uma consulta com um maior tempo de atendimento, atinge-se o resultado esperado.

No presente caso clínico, foi utilizado o peróxido de hidrogênio a 35\%, com um resultado altamente satisfatório, trazendo ao paciente uma estética do sorriso muito agradável, com uma mudança de cor bastante significativa, que de acordo com a escala VitaPan, foi da coloração A3 para A1. Corroborando com os achados no estudo de Prado e Sartori ${ }^{2}$, que fizeram um clareamento de dentes vitais amarelados em um paciente através do uso de peróxido de hidrogênio a 35\% em ambas as arcadas com aplicação subsequente de flúor neutro em três sessões, com intervalos de sete dias entre cada sessão. Concluiu-se que a técnica é simples e eficiente, não promoveu dano aos tecidos moles e duros da cavidade bucal, além do resultado final ser satisfatório.

A hipersensibilidade dentinária é um dos principais efeitos negativos do clareamento dental, ocorrendo com maior frequência no clareamento em consultório devido à maior concentração do produto ${ }^{11}$. Geralmente persiste por um ou dois dias após a sessão, e pode variar de leve e moderada, à severa ${ }^{12}$. Esta sensibilidade se deve a capacidade dos agentes clareadores de penetrar no esmalte e na dentina devido à alta permeabilidade destas estruturas dentais atingindo diretamente a polpa ${ }^{13}$.

Uma pesquisa realizada por Silva et al. ${ }^{14}$, com 0 objetivo estudar a sensibilidade dentinária frente ao uso de fonte luminosa associada ao clareamento de consultório, concluiu-se que o uso do laser infravermelho após cada sessão de clareamento dental não proporcionou redução na sensibilidade dentinária.

Gomes ${ }^{13}$ e Soares et al. ${ }^{4}$ afirmam que o grau de hipersensibilidade no clareamento em consultório é maior do que o clareamento caseiro, já que o Peróxido de Hidrogênio atinge a polpa de forma mais fácil por estar mais concentrado.

Penha et al. $^{15}$ evidenciam que no clareamento dental o princípio ativo do peróxido de hidrogénio $35 \%$ é de fato responsável pela alteração de cor e presença de sensibilidade dentária. Desta forma, em seu estudo, o paciente foi convidado a responder um questionário Wong e Baker $^{6}$ para avaliar o índice de sensibilidade após as sessões. E segundo a escala de classificação de sensibilidade adotada, o paciente relatou que ao falar e executar respiração bucal sentia sensibilidade. $E$ em relação ao nível da hipersensibilidade após as sessões, o maior score foi 6 , significando que "dói muito".

Jesus $^{16}$ aponta que a sensibilidade dental após o tratamento clareador está associada ao tempo de exposição do agente clareador ao dente, a escolha do peróxido a ser utilizado, ao processo de difusão dos peróxidos na estrutura dental e também à concentração do agente clareador.

Para Leornard et al. ${ }^{17}$, a possibilidade de dor advinda do clareamento pode ser considerada como fator limitante para a indicação desta técnica cosmética naqueles pacientes em que há diagnóstico de hipersensibilidade dentinária pré-existente. Porém, ao longo do tempo, técnicas e materiais clareadores que visam a redução da sensibilidade vêm demonstrando bons resultados clínicos.

Existem diversas alternativas para tratar a sensibilidade durante e após o clareamento, Gomes $^{13}$ inclui várias opções de tratamento e/ou associações delas, como: Redução do agente clareador, uso de soluções remineralizadoras (flúor) e soluções dessensibilizantes, nitrato de potássio, terapia de laser de baixa potência, analgésicos e antiinflamatórios.

Por outro lado, discordando de Gomes ${ }^{13}$, Palomino $^{18}$ afirma que, apesar da grande quantidade de agentes remineralizadores e dessensibilizantes disponíveis, a eficiência, rapidez de atuação e duração destes tratamentos está longe do ideal, uma vez que muitos são constituídos por finas películas, facilmente removidas em procedimentos 
rotineiros como escovação dental e mastigação ou não aderem às paredes dos túbulos dentinários ou ainda, agem apenas inibindo a propagação do estimulo da dor, sem regenerar a superfície dental.

Oliveira et al. ${ }^{19}$ relatam em seu caso que, - clareamento dentário realizado em sua paciente aliviou seu desconforto, proporcionando-Ihe satisfação interna, qualificando a relação consigo mesma e com os outros e elevando sua autoestima. Silva ${ }^{7}$ relata em seu trabalho que as pessoas em geral, buscam dentes cada vez mais brancos podendo ultrapassar o aspecto de naturalidade.

Neste trabalho, foi aplicado um questionário de satisfação do indivíduo em relação à estética do próprio sorriso ${ }^{7}$. Assim, foi possível observar um ótimo grau de satisfação do paciente em relação ao seu sorriso, comparando com antes do procedimento. Os resultados obtidos após essa avaliação mostram que o paciente ficou com uma maior autoestima em relação ao seu sorriso, comparado com anterior ao clareamento dental.

Silva ${ }^{7}$ mostra em seu estudo que dos 55 pacientes que responderam ao questionário de grau de satisfação, aplicado em sua pesquisa, $89 \%$ gostariam de mudar a cor dos dentes, $80 \%$ mudariam a forma e o tamanho dos dentes, $75 \%$ gostariam de mudar algo relacionado à disposição dos seus dentes e 53\% mudariam algo relacionado ao periodonto. Podemos observar, então, que a cor dos dentes é citada como um dos principais tratamentos para a melhora da estética do sorriso.

\section{CONCLUSÃO}

O clareamento dental é um procedimento estético simples, pouco invasivo, que traz ao paciente resultados estéticos satisfatórios. Após as sessões de clareamento, o paciente obteve um ótimo resultado, levando o seu sorriso de coloração A3 para A1 de acordo com a escala Vita (Vitapan® Classical). Em relação ao grau de desconforto de sensibilidade após as sessões, foi caracterizando como um desconforto que dói muito, após a segunda sessão, mas perdurando apenas nas primeiras $24 \mathrm{~h}$, todavia, um alto nível de satisfação depois do tratamento foi obtido.

\section{REFERÊNCIAS}

1. Carvalho EM, Silva AS, Costa JF, Firoozmand LM, Silva BMAH, Lago ADN. Uso da luz no clareamento dental em consultório: há controvérsias? Rev Pesq Saúde. 2015;16(1):189-93.
2. Prado HD, Sartori LA. Clareamento de dentes vitais amarelados. Rev Naval Odontol. 2010;3(3):5-10.

3. Pasquali EL, Bertazzo CA, Anziliero L. Estudo dos efeitos do clareamento dental sobre o esmalte: uma revisão das evidências para a indicação clínica. Perspectiva Erechim. 2014;38(104):99-108.

4. Soares FF, Sousa JAC, Maia CC, Fontes CM, Cunha LG, Freitas AP. Clareamento em dentes vitais: uma revisão literária. Saúde.Com. 2008;4(1):72-84.

5. Fonseca EE, Silva EROI. Clareamento em dentes vitais [monografia]. Pindamonhangaba: Faculdade de Pindamonhangaba; 2017.

6. Wong DL, Baker CM. Smiling faces as anchor for pain intensity scales. Pain. 2001;89(2-3):295-300.

7. Fonseca AS. Odontologia estética: a arte da perfeição. São Paulo: Artes Médicas; 2008.

8. Roberto AR, Jassé FF, Boaventura JMC, Martinez TC, Rastelli ANS, Oliveira Júnior OB et al. Evaluation of tooth color after bleaching with and without light-activation. Rev odonto ciênc. 2011; 26(3),247-52.

9. Barbosa DC, De'Stefani TP, Ceretta LB, Ceretta RA, Simões PW, D'Altoé LF. Estudo comparativo entre as técnicas de clareamento dental em consultório e clareamento dental caseiro supervisionado em dentes vitais: uma revisão de literatura. Rev odontol Univ Cid São Paulo. 2017; 27(3):244-52.

10. Ferreira DO, Azevedo JF, Cavalcante AN. Estudo da dessensibilização prévia ao clareamento dental em consultório: uma revisão de literatura. RFO UFBA. 2014;44(1):49-56.

11. Godoy CEM. Efeito de dois agentes dessensibilizantes sobre o grau de clareamento e sensibilidade da dentina, aplicados previamente ao clareamento de consultório-ensaio clínico controlado, randomizado, duplo-cego [dissertação]. Cascavel: Unioeste; 2016.

12. Gomes AMRT. Sensibilidade pósclareamento: revisão de literatura [monografia]. Campina Grande; UEPB; 2014.

13. Silva LVF, Silva ARJ, Costa Figueiredo CHM, Penha ES, Guênes, GMT. Efeito do laser infravermelho como terapia para sensibilidade dentinária decorrente de clareamento dental de consultório. Arch Health Invest. 2018;7(Spec Iss 7):48.

14.Penha ES, Pinto WT, Santos RL, Guênes GMT, de Medeiros LA, Lima AMA. Avaliação 
de diferentes sistemas de clareamento dental de consultório. RFO UPF. 2015;20(3):281-86.

15.Jesus $A B$. Sensibilidade dental após 0 tratamento clareador [monografia]. Londrina: Universidade Estadual de Londrina; 2017.

16. Leonard JR, Ralph H, Smith LR, Garland GE, Tiwana KK, Zaidel LA et al. Evaluation of side effects and patients' perceptions during tooth bleaching. J Esthet Restor Dent. 2007;19(6):355-64.

17.Palomino K. Avaliação de agentes remineralizadores e dessensibilizantes no tratamento de clareamento dental: estudo in vitro [tese]. Ribeirão Preto: Universidade de São Paulo, USP; 2013.

18. Oliveira JAG, Cunha VDPP, Fajardo RS, Rezende MCRA. Clareamento dentário $x$ autoestima $x$ autoimagem. Arch Health Invest. 2014;3(2):21-25.

\section{CONFLITO DE INTERESSES}

Os autores declaram não haver conflitos de interesse

\section{AUTOR PARA CORRESPONDÊNCIA}

\section{José Henrique de Araújo Cruz}

Rua Paulo Diogenes, 57 - Bairro Centro, 59990000. Rafael Fernandes - RN, Brasil Telefone: (83) 99625-0125

E-mail: henrique_araujo1992@hotmail.com 